\title{
Tunable aptamer capillary electrophoresis and its application to protein analysis
}

Hongquan Zhang, Xing-Fang Li and X. Chris Le*

\section{Supporting Information}

\section{Materials and Methods}

Human IgE, HIV-1 RT, thrombin, PDGF-BB, and PDGF-AB were obtained from Athens Research \& Technology (Athens, GA), Worthington Biochemical (Lakewood, NJ), Haematologic Technologies (Essex Junction, VT), and R\&D Systems (Minneapolis, MN), respectively. Aptamers were synthesized by Integrated DNA Technologies (Coralville, IA). They were fluorescently labeled at the 5' end with FAM (carboxyfluorescein). Aptamer sequences (both full-length and truncated) are listed in Supplemental Table 1. Tris-glycine (TG) buffer (25 mM Tris and $192 \mathrm{mM}$ glycine, $\mathrm{pH}$ 8.3) was diluted with deionized water from 10×TG buffer (from Bio-Rad Laboratories, Mississauga, ON, Canada) and was then adjusted to $\mathrm{pH} 8.5$ by $1 \mathrm{M}$ $\mathrm{NaOH}$.

We have optimized the conditions for binding, separation, and detection, maximizing the sensitivity and speed of analysis. These optimized parameters include the incubation temperature and time, the separation buffer conditions, and the effects of $\mathrm{Mg}^{2+}$ on the formation of specific protein-aptamer complexes.

For calibration, IgE, HIV-RT, thrombin, and PDGF-BB (each 1-100 nM) were incubated at $37^{\circ} \mathrm{C}$ for 10 min with $300 \mathrm{nM}$ fluorescently labeled aptamer for PDGF-BB and $200 \mathrm{nM}$ fluorescently labeled aptamers for other specific proteins. The incubation buffer contained $20 \mathrm{mM}$ Tris. $\mathrm{HCl}$ and $1 \mathrm{mM} \mathrm{MgCl}_{2}$ at $\mathrm{pH}$ 7.4. A laboratory-built capillary electrophoresis laser induced fluorescence system ${ }^{[10]}$ was 
used for the analysis of the multiple protein-aptamer complexes. Uncoated fusedsilica capillaries $(20 \mu \mathrm{m}$ i.d., $150 \mu \mathrm{m}$ o.d, $40 \mathrm{~cm}$ in length, Polymicro Technologies, Phoenix, AZ) were used for separation. Electrophoresis was carried out at room temperature with a voltage of $18 \mathrm{kV}$ (electric field $450 \mathrm{~V} / \mathrm{cm}$ ) and Tris-glycine buffer $(\mathrm{pH} 8.5)$ as the running buffer. Samples were injected electrokinetically for $5 \mathrm{~s}$ at 18 $\mathrm{kV}$. Following each electrophoresis run, the capillary was washed sequentially with $20 \mathrm{mM} \mathrm{NaOH}$ (5 min), water (2 min), and Tris-glycine buffer (5 min). A blue argon ion laser (488 nm) was used for excitation, and fluorescence was detected at $515 \mathrm{~nm}$.

Human serum was obtained from Sigma-Aldrich (Oakville, ON, Canada) and was diluted 10 times with incubation buffer. The dilute serum was spiked with $25 \mathrm{nM}$ each of IgE, HIV-RT, thrombin, and PDGF-BB. An aliquot $(50 \mu \mathrm{L})$ of the sample was incubated at $37^{\circ} \mathrm{C}$ for $10 \mathrm{~min}$ with $100 \mathrm{nM}$ of four fluorescently labeled aptamers for the specific proteins and another $4 \mu \mathrm{M}$ of a non-specific, non-fluorescent 49 -mer oligonucleotide. The non-specific oligonucleotide was used to reduce interference from the serum matrix.

[10]. (a) Wan, Q.-H.; Le, X.C. Anal. Chem. 2000, 72, 5583-5589. (b) Wang, H.; Lu, M.; Mei, N.; Lee, J.; Weinfeld, M.; Le, X.C. Anal. Chim. Acta 2003, 500, 13-20. (c) Wang, H. Lu, M.; Le, X.C. Anal. Chem. 2005, 77, 4985-4990. 
Supplemental Table 1. Summary of proteins and their aptamers used in this study

\begin{tabular}{|c|c|c|c|c|}
\hline Proteins & $\begin{array}{l}\text { Mol. } \\
\text { Wt. } \\
\text { (kDa) }\end{array}$ & $\mathrm{pl}$ & $\begin{array}{l}\text { Aptamer } \\
\text { size }\end{array}$ & Aptamer sequence \\
\hline \multirow[t]{2}{*}{$\operatorname{lgE}$} & 200 & $\sim 9.0$ & $38 \mathrm{nt}$ & $\begin{array}{l}\text { TGGGGCACGTTTATCCGTCCCTCCTAGTGGC } \\
\text { GTGCCCC }\end{array}$ \\
\hline & & & $53 \mathrm{nt}$ & $\begin{array}{l}\text { AGGGGCACGTTTATCCGTCCCTCCTAGTGGC } \\
\text { GTGCCCCTGTCTGACTGTCTCG }\end{array}$ \\
\hline \multirow[t]{3}{*}{ HIV-RT } & 120 & $\sim 8.2$ & $49 \mathrm{nt}$ & $\begin{array}{l}\text { ATCCGCCTGATTAGCGATACTCAGAAGGATA } \\
\text { AACTGTCCAGAACTTGGA }\end{array}$ \\
\hline & & & $80 \mathrm{nt}$ & $\begin{array}{l}\text { ATCCGCCTGATTAGCGATACTCAGAAGGATA } \\
\text { AACTGTCCAGAACTTGGAACTCATCACTACAA } \\
\text { TACATCATACTTCACTA }\end{array}$ \\
\hline & & & $2 \times 81 \mathrm{nt}$ & $\begin{array}{l}\text { ATCCGCCTGATTAGCGATACTTACGTGAGCGT } \\
\text { GCTGTCCCCTAAAGGTGATACGTCACTTGAG } \\
\text { CAAAATCACCTGCAGGGG }\end{array}$ \\
\hline \multirow[t]{2}{*}{ Thrombin } & 36 & $\begin{array}{l}6.35- \\
7.6\end{array}$ & $38 \mathrm{nt}$ & $\begin{array}{l}\text { CAGTCCGTGGTAGGGCAGGTTGGGGTGACTT } \\
\text { CGTGGAA }\end{array}$ \\
\hline & & & $76 \mathrm{nt}$ & $\begin{array}{l}\text { AGATGCCTGTCGAGCATGCTCTTTGGAGACA } \\
\text { GTCCGTG } \\
\text { GTAGGGCAGGTTGGGGTGACTTCGTGGAAG } \\
\text { AAGCGAGA }\end{array}$ \\
\hline PDGF-BB & 25 & $\begin{array}{l}9.5- \\
10.5\end{array}$ & $2 \times 33 n t$ & $\begin{array}{l}\text { TGGGAGGGCGCGTTCTTCGTGGTTACTTTTAGT } \\
\text { CCCG }\end{array}$ \\
\hline PDGF-AB & 27 & $\begin{array}{l}9.5- \\
10.5\end{array}$ & $33 \mathrm{nt}$ & AGGGCGCGTTCTTCGTGGTTACTTTTAGTCCCG \\
\hline $\begin{array}{l}\text { Non- } \\
\text { specific }\end{array}$ & & & $49 \mathrm{nt}$ & $\begin{array}{l}\text { TGGTCTTGTGTGGCTGTGGCTATGTCTGATCTTA } \\
\text { ATCCACGAAGTCACC }\end{array}$ \\
\hline & & & & \\
\hline
\end{tabular}

